\title{
Integration of Keyword and Feature Based Search for Image Retrieval Applications
}

\author{
A. Vadivel $^{1}$, Shamik Sural ${ }^{2}$, and A.K. Majumdar ${ }^{1}$ \\ ${ }^{1}$ Department of Computer Science and Engineering, Indian Institute of Technology, \\ Kharagpur 721302, India \\ \{vadi@cc, akmj@cse\}.iitkgp.ernet.in \\ ${ }^{2}$ School of Information Technology, Indian Institute of Technology, \\ Kharagpur 721302, India \\ shamik@sit.iitkgp.ernet.in
}

\begin{abstract}
The main obstacle in realizing semantic-based image retrieval is from the web that semantic description of an image is difficult to capture in low-level features. Text based keywords can be generated from web documents to capture semantic information narrowing down the search space. We use an effective dynamic approach to integrate keywords and color-texture features to take advantage of their complementing strengths. Experimental results show that the integrated approach has better retrieval performance than both the text based and the content-based techniques.
\end{abstract}

\section{Introduction}

Images are very frequently embedded in HTML documents to express the intended message effectively on the World Wide Web [16] (WWW). Conventional search engines use only keyword based search for retrieval and often takes many iterations to converge. Conventional search engines do not integrate low-level features to support image retrieval queries. On the other hand, a large number of content-based image retrieval systems have been developed in the academia and the industry $[4,5,8,12]$ which use low-level features such as color, texture and shape. These approaches do not include keyword based textual information to complement the retrieval and narrow the search space. As result, precision of retrieval for general-purpose image retrieval applications tends to be very low. On the other hand text based retrievals like Google attend reasonably high precision. We feel that an effective image retrieval system should make use of both keywords and low-level features of an image and try to integrate keyword-based and content-based image retrieval techniques as a single system. Keywords-based capture high-level semantics but they cannot depict complicated image features very well. The key word extraction process also should be made dynamic to be really useful. On the other hand, content-based techniques can capture low-level image features and can accept images as queries. An image query process is hard to start, as the user has to specify a query image by selecting an existing image and the user normally does not have access to all the images, which can be used as queries. It is well established that while uploading images, one cannot possibly label or tag it with test. The reason is that, such labeling is subjective, often 
incomplete and heavily manpower intensive. On the other hand, it is not a difficult properties to include keywords along with sample images while preparing a query. Essentially, the user not only specifies a query image, but also mentions in text form, what is the semantic content of the image that he is interested in an information not captured just by the low-level features. In our system users can start their search process by providing keywords. A set of images are retrieved from the database and displayed to the user based on the keyword. From these initial results, user can further select image either as content-based queries or as content-based supplemented with keywords to narrow down the search space. The final retrieved images are based on combined scores of the keyword-based and the content-based searching. We have built a web-based application [13] which is available in the public domain for interested users to repeat our experiments and perform the searches.

In the next section, we explain our method for keyword-based indexing and retrieval of images. In section 3, we explain content-based image indexing and retrieval using COLTEX a color-texture retrieval technique. We describe the combined keyword and COLTEX based retrieval procedure in section 4. Experimental results are included in section 5 and we draw conclusions in the last section of the paper.

\section{Keyword-Based Indexing and Retrieval of Images}

Keyword-based image retrieval can be based on the traditional text information retrieval (IR) technique [7]. However, to improve retrieval performance, we should make use of the structure of HTML documents. This is because the words or the terms appearing at different locations of an HTML document have different levels of importance or relevance to related images [2]. Therefore, we need to assign term weights based on term positions. In our proposed approach, we extract texts around the images and calculate occurrence frequencies of the words in the text. The stop words are filtered out and others are stored in a database. Figure 1, shows the keyword based search interface and a typical output of the web-based application. In our database, a given image may be related to many keywords and a single keyword is related to many images. If a single keyword is provided as query string, the search space is wide and returned images may be from different categories. To minimize the search space, more than one keywords can be provided for performing the search.

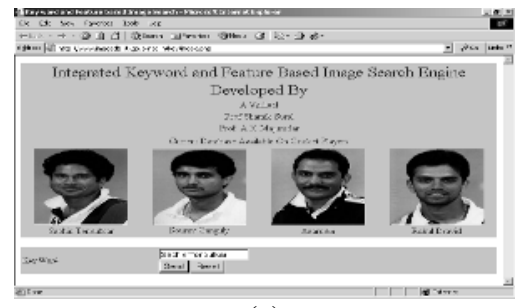

(a)

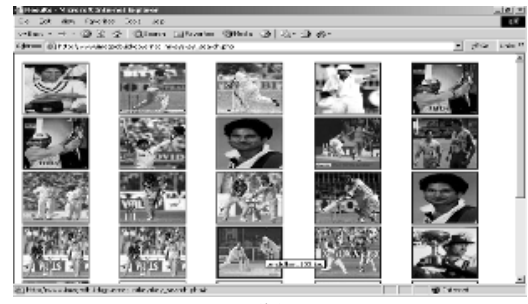

(b)

Fig. 1. Web - Based Image Retrieval System (a) Interface to perform keyword-based search (b) Results of a keyword-based search 


\section{Color-Texture Feature Based Indexing and Retrieval of Images}

We use a combined color and texture feature called COLTEX for performing lowlevel image indexing and retrieval. While the method has been presented in [11], we give a brief overview here. COLTEX describes color and texture content of the images and is represented as a color texture matrix CTM where

$$
C T M=\left(\begin{array}{ll}
C T M_{C C} & C T M_{C G} \\
C T M_{G C} & C T M_{G G}
\end{array}\right)
$$

Each component of the COLTEX matrix $C T M_{a b}, a, b \in\{C, G\}$ is a sub matrix represents the combinations of color-color, color-texture, texture-color and texturetexture, respectively. This feature vector is based on human visual perception of the color and texture of an image pixel and its performance has been found to be encouraging [10]. Each sub matrix of COLTEX is given by

$$
\begin{aligned}
& C T M_{C C}=\operatorname{ctm}_{C C}(i, j) \mid i=0 \ldots C_{L}, j=0 \ldots C_{L} \\
& C T M_{C G}=\operatorname{ctm}_{C G}(i, j) \mid i=0 \ldots C_{L}, j=0 \ldots G_{L} \\
& C T M_{G C}=\operatorname{ctm}_{G C}(i, j) \mid i=0 \ldots G_{L}, j=0 \ldots C_{L} \\
& C T M_{G G}=\operatorname{ctm}_{G G}(i, j) \mid i=0 \ldots G_{L}, j=0 \ldots G_{L}
\end{aligned}
$$

The values of $C_{L}$ and $G_{L}$ are as follows

$$
\begin{aligned}
& C_{L}=\frac{2 \pi}{Q_{H}}+1 \\
& G_{L}=\frac{255}{Q_{I}}+1
\end{aligned}
$$

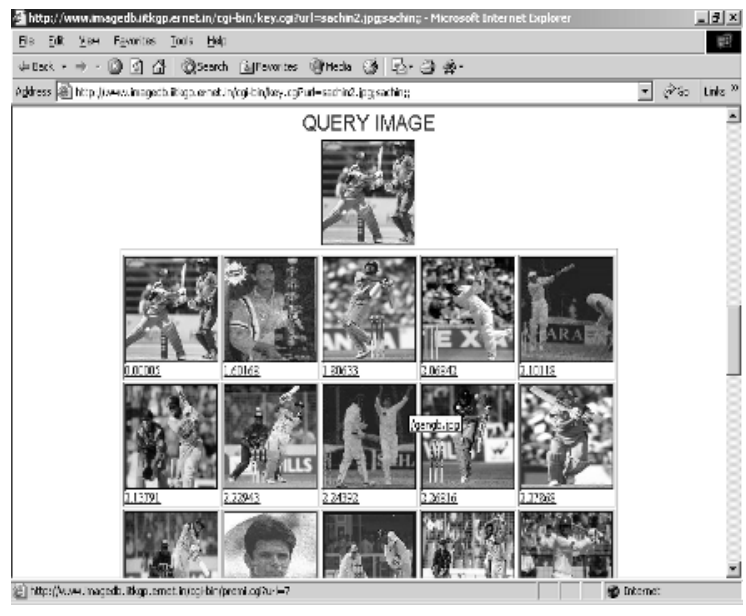

Fig. 2. Retrieval based on COLTEX 
where $Q_{I}$ and $Q_{H}$ are the quantization values for color and texture respectively. $2 \pi$ is the domain values for the hue and 255 is the maximum intensity level in the HSV color space [10].

For each image crawled from the web the COLTEX matrix is extracted and stored in a database. During image retrieval, COLTEX is extracted from the query image and compared with the all the features in the database. The Manhattan Distance is used to measure the similarity between COLTEX of the query image and each of the images in the database. Images with a distance smaller than a pre-defined threshold are retrieved from the database and presented to the user. Figure 2 shows the interface and retrieval results of feature-based extraction.

\section{Image Retrieval by Combining Keywords and COLTEX}

We combine keyword and COLTEX based search by the use of an input keyword and an image of interest from the set of images initially displayed. The keyword based retrieval is first applied based on the input keyword and COLTEX features of the retrieved images are extracted and compared with the database images to return the final set of retrieved images as shown in figure 3. The number of retrieved images depends on the search space. The result shows the final retrieved images and their distance values. If two images are close to each other in terms of their content the distance value is as close to zero. The distance value is more for images with different semantic content content.

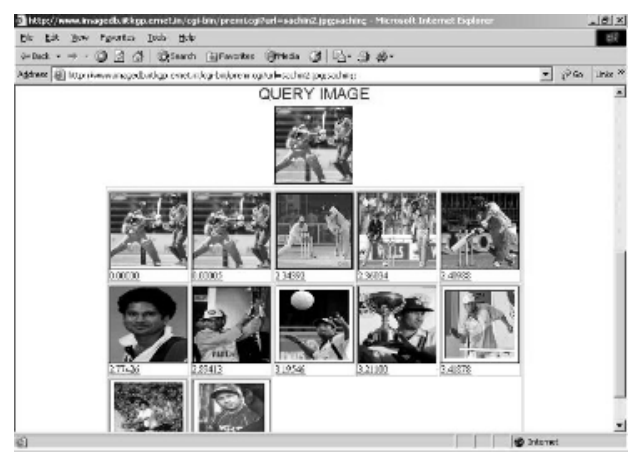

Fig. 3. Image retrieval based on both keyword and COLTEX

\section{Results}

In this section, we show results of keyword-based, COLTEX-based and combined keyword and COLTEX based retrieval. Table 1 shows the average precision $(\mathrm{P})$ in percentage for nearest neighbors 10, 20, 50 and 100 . 
Table 1. Precision of retrieval

\begin{tabular}{|l|c|c|c|c|}
\hline Features & $\begin{array}{c}\mathbf{P}(\mathbf{1 0}) \\
\mathbf{\%}\end{array}$ & $\begin{array}{c}\mathbf{P}(\mathbf{2 0}) \\
\mathbf{\%}\end{array}$ & $\begin{array}{c}\mathbf{P}(\mathbf{5 0}) \\
\mathbf{\%}\end{array}$ & $\mathbf{P}(\mathbf{1 0 0}) \boldsymbol{\%}$ \\
\hline Keyword based & 46.00 & 35.90 & 25.40 & 19.98 \\
\hline COLTEX & 55.00 & 46.90 & 35.40 & 22.98 \\
\hline COLTEX and Keyword combined & $\mathbf{9 2 . 3 3}$ & $\mathbf{8 7 . 2 8}$ & $\mathbf{6 4 . 2 1}$ & $\mathbf{5 1 . 0 0}$ \\
\hline
\end{tabular}

We have used a large number of randomly selected images of different categories and content and calculated the average precision. Form the results, it is observed that the performance of the combined method is quite encouraging. We have earlier shown that COLTEX performance is better than other color-texture based methods. Hence, it is expected that the combined method have better performance compared to the existing approaches.

\section{Conclusions}

We have studied the important role of textual keywords in describing high-level semantics of an image. We have also described The COLTEX a feature represents color-texture content of images that can be used for image retrieval. We have combined both the high level and low level features effectively to achieve very high average precision of retrieval. An image retrieval system has been developed to demonstrate our work and interested readers are encouraged to use our system. Promising retrieval results are obtained from our web based system. We plan to increase the database size to about 10,000 images and compare our results with other keyword and low-level feature based retrieval systems [1,3,6,9,14,15].

\section{Acknowledgement}

The work done by Shamik Sural is supported by research grants from the Department of Science and Technology, India, under Grant No. SR/FTP/ETA-20/2003 and by a grant from IIT Kharagpur under ISIRD scheme No. IIT/SRIC/ISIRD/2002-2003. Work done by A. K. Majumdar is supported by a research grant from the Department of Science and Technology, India, under Grant No. SR/S3/EECE/024/2003-SERCEngg.

\section{References}

1. Hyoung K. Lee and Suk I. Yoo: Nonlinear Combining of Heterogeneous Features in Content-Based Image Retrieval. International journal on Computer research, Volume 11, No. 3, (2002)

2. Lempel.R and A.Soffer: PicASHOW: Pictorial Authority Search by Hyperlinks on the Web. WWW10, May 1-5, Hong Kong, (2001). 
3. Mezaris .V, et. al. : Combining Textual and Visual Information Processing for Interactive Video Retrieval: SCHEMA's participation in TRECVID 2004. TRECVID 2004. Text Retrieval Conference TRECVID Workshop, Gaithersburg, Maryland, 15-16 November (2004)

4. Niblack W. et al: The QBIC Project: Querying Images by Content using Color Texture and Shape. SPIE Int. Soc. Opt. Eng., In Storage and Retrieval for Image and Video Databases, Vol. 1908, (1993) 173-187

5. Ortega, M. et al: Supporting Ranked Boolean Similarity Queries in MARS. IEEE Trans. on Knowledge and Data Engineering, Vol. 10 (1998) 905-925

6. Pentland P.A., R. W. Picard, and S. Sclaroff : Photobook: Content-based Manipulation of Image Databases. Int. Journal of Computer Vision, Vol. 18, No. 3, (1996) 233--254, (1996)

7. Salton G.: Introduction to Modern Information Retrieval, McGraw-Hill Book Company. (1993).

8. Smith, J.R., Chang, S.-F.: VisualSeek: A Fully Automated Content based Image Query System. ACM Multimedia Conf., Boston, MA (1996)

9. Srihari.R.K: Combining text and image information in content-based retrieval. International Conference on Image Processing (Vol. 1)-Volume 1 October 23 - 26, (1995)

10. Vadivel.A, Shamik Sural and A.K.Majumdar: Human color perception in the HSV space and its application in histogram generation for image retrieval. International Conference on Color Imaging X: Processing, Hardcopy, and Applications, part of the IS\&T/SPIE Symposium on Electronic Imaging, December (2005)

11. Vadivel.A, Shamik Sural and A.K.Majumdar: Color-Texture Feature Extraction using Soft Decision from the HSV Color Space", International Symposium on Intelligent Multimedia Processing, Hong Kong, 2004

12. Wang, J.Z., Li, J., Wiederhold, G.: SIMPLIcity: Semantics-sensitive Integrated Matching for Picture Libraries. IEEE Trans. on PAMI, Vol. 23 (2001).

13. Web Based On Line System: Http://www.imagedb.iitkgp.ernet.in/key/index.php.

14. Xiang Sean Zhou Huang, T.S.: Unifying keywords and visual contents in image retrieval. IEEE Multimedia Volume 9, Issue 2, 22-33, (April 2002)

15. Yixin Chen, James Z. Wang and Robert Krovetz : CLUE: Cluster-based Retrieval of Images by Unsupervised Learning. IEEE Transactions on Image Processing, vol. 14, 15 (2005) in press.

16. Zhuge. H. VEGA-KG: A Way to the Knowledge Web. In Proc. of $11^{\text {th }}$ International World Wide Web Conference (WWW2002), May, Honolulu, Hawaii, USA, (2002). 